\title{
LA GESTIÓN DE LA CALIDAD DE LA ENSEÑANZA EN EL MARCO DE UN SISTEMA DE FORMACIÓN INICIAL DOCENTE: ESTUDIO DE CASO
}

\author{
Pedro Enrique Rosales Villarroel
}

\begin{abstract}
Resumen
La investigación se enmarca en el propósito de describir y reconocer desde la perspectiva de los actores el carácter particular de la gestión de la enseñanza en la Escuela de Pedagogía en Historia y Geografía de la Universidad Autónoma de Chile, y en sus relaciones con las demandas surgidas del contexto situacional y de las definiciones internas articuladas en el Proyecto Educativo Institucional. La orientación básica de este estudio se inscribe en el campo temático de la Teoría de la Organización Educativa, el cual se caracteriza por su gran amplitud y naturaleza multidisciplinar. Más concretamente, esta investigación se basa en las aportaciones que provienen especialmente del enfoque actual de la calidad. Este trabajo se nutre de los fundamentos teóricos que provienen del campo temático antes mencionado y de las nuevas perspectivas asociadas a la Formación Inicial de Profesores (FIP). En este contexto la FIP descansa en un entramado organizativo cuyos niveles básicos de análisis son los siguientes: individuo, grupo y organización, elementos claves para interpretar y mejorar con acierto la naturaleza particular de las dinámicas microorganizativas que se despliegan en el contexto de la formación.

Palabras claves: Calidad de la enseñanza, formación inicial docente, organización escolar
\end{abstract}

\section{A GESTÃO DA QUALIDADE DO ENSINO NO MARCO DE UM SISTEMA DE FORMAÇÃO INICIAL DOCENTE: ESTUDO DE CASO}

\begin{abstract}
Resumo
A investigação teve como objetivo descrever e reconhecer, a partir da perspectiva dos atores, a natureza particular da gestão da educação na Escola de Educação em História e Geografia da Universidade Autônoma do Chile, nas suas relações com as demandas decorrentes do contexto situacional e definições internas articuladas no Projeto Institucional de Educação. A orientação básica deste estudo se insere no campo da Teoria da Organização Educacional, caracterizado pela sua grande amplitude e natureza multidisciplinar. Mais especificamente, está pesquisa é baseada em aportes provenientes sobretudo da atual abordagem para a qualidade. O trabalho baseia-se nos fundamentos teóricos que estão sob a área temática acima e novas perspectivas associadas à formação inicial de professores (FIP). Neste contexto, a FIP é baseada em uma estrutura organizacional que tem como base os seguintes níveis de análise: individual, grupal e organizacional, elementos-chave para interpretar corretamente e melhorar a especificidade da dinâmica micro-organizativa que é desenvolvida no contexto da formação. Palavras-chave: Qualidade do ensino, formação inicial docente, organização escolar.
\end{abstract}




\section{P. E. Rosales Villarroel}

\section{Introducción}

La investigación se enmarca en el propósito de describir y reconocer desde la perspectiva de los actores el carácter el carácter particular de la gestión de la enseñanza en la Escuela de Pedagogía en Historia y Geografía de la Universidad Autónoma de Chile, y en sus relaciones con las demandas surgidas del contexto situacional y de las definiciones internas articuladas en el Proyecto Educativo Institucional. La mayor parte de las investigaciones desarrolladas sobre el cambio en las organizaciones educativas muestra que las organizaciones convencionales y burocráticas no se desenvuelven bien en las inestables condiciones de la postmodernidad. Los tipos de organizaciones con más probabilidades de prosperar en el mundo postmoderno son las caracterizadas por la flexibilidad, la adaptabilidad, la creatividad, el aprovechamiento de las oportunidades, la colaboración, el perfeccionamiento continuo, una orientación positiva hacia la resolución de problemas y el compromiso para maximizar su capacidad de aprender sobre su ambiente y sobre ellas mismas. (LEINBERGER Y TUCKER, 1991).

Lo anterior supone una capacidad permanente de cambio, de innovación y mejora. Esto obliga a afectar la dinámica básica de toda organización como es la tendencia a la estabilidad. Todo cambio en profundidad en una organización implica un cambio en su cultura, en las creencias y los valores que la definen y sustentan.

La era del conocimiento en que vivimos presenta a las sociedades modernas desafíos sin precedentes históricos. Los vertiginosos avances en tecnología y las comunicaciones instantáneas que aceleran el proceso de globalización y acercan a la gente y los países como jamás había sucedido, causan profundo impacto en la educación.

Inmediatamente surge la pregunta, ¿cómo vamos a educar a nuestros alumnos en un mundo como el que nos toca vivir? Y la respuesta tiene hoy tanto de desafío y potencial de problema, como de oportunidad. Los desafíos que enfrenta la educación son enormes, razón por la cual adquiere una alta prioridad en el desarrollo de los países, las sociedades, las organizaciones.

Surge así, de esta manera, una organización que tiene fundamento en la información y el conocimiento y que debe concentrarse en nuevas prioridades congruentes con el grado de desarrollo de la sociedad. Las características de la nueva organización se traducen en:

* Énfasis en el ser humano como creador y gestor de información y 
conocimiento.

* Necesidad de desarrollar capital humano.

* El ser humano como centro de la organización.

* Énfasis en la calidad.

* Énfasis en el desarrollo integral de la persona.

* Necesidad de desarrollo integral en la organización.

* Énfasis en las comunicaciones formales e informales.

* Gestión basada en la colaboración e integración.

* Estilo de liderazgo participativo basado en el respeto por las personas.

* Estructura matricial de organización.

En ese contexto, que define la naturaleza particular de las demandas que se hacen al sistema educativo, el proceso evaluación y orientación hacia la calidad aparece como uno de los elementos que permite a los establecimientos hacer uso de su autonomía, por lo tanto es muy importante tener claridad respecto al significado de estos conceptos, así como de los enfoques que existen al respecto.

- En base a los criterios precedentes se define una estructura de trabajo que busca un acercamiento al carácter y el sentido de la Evaluación de la Calidad Educativa, para iniciar a partir de ella un proceso de reforma continua a las organizaciones escolares, en aras a hacer correspondientes las tareas formativas de la escuela como el cúmulo de demandas sociales y culturales que a ésta se le hacen.

En ese mismo contexto el tema de la calidad de la educación se abre a la discusión. La educación no sólo se apropia de la Economía del término eficiencia para estimar la optimización que hace el sistema de los recursos disponibles, sino que además aplica el término eficacia para plantearse el grado de cumplimiento del sistema educacional respecto de los objetivos propuestos. De esta manera el sistema no solamente se interesa en asegurarse cuántos ingresan, cuántos son retenidos, fluyen y son promovidos de un grado a otro hasta egresar, sino se interesa en saber cuánto aprenden los educandos en su paso por el sistema, qué aspectos afectan en el aprendizaje, qué satisfacción tiene la sociedad con el servicio que presta.

Entonces la calidad tiene una cara visible que se traduce en un resultado o también denominado niveles de logros alcanzados por los/las escolares en diferentes grados y niveles del sistema educacional, en sus diferentes áreas curriculares, especialmente lecto-escritura y matemática, por 


\section{P. E. Rosales Villarroel}

tratarse de herramientas instrumentales sobre las cuales se construye cualquier otro aprendizaje.

Sin duda ese "producto escolar" es afectado por múltiples aspectos denominados factores asociables al rendimiento escolar que subyacen afectándolo positiva o negativamente ya sea en el contexto comunitario en el que se inscribe la escuela, ya como insumos educacionales y humanos, ya en los procesos técnico pedagógicos que tienen su expresión en el aula.

Para juzgar la calidad de cada uno de ellos y plantearse perspectivas de mejoramiento es necesario abrir espacios de discusión entre los diferentes grupos representativos de la sociedad, porque cada elemento supone un atributo al que se le asigna valor. Quien asigne valor a algo (objeto, situación, servicio u otro), implícitamente compara con un referente social y cultural construido históricamente en su vida personal. Al hablar de calidad se hace referencia al ser y al asignarle valor a la calidad se está asignando una cualidad que se encuentra en el plano del estar, de esta manera se tiene que la calidad es substantiva y la cualidad es adjetiva.

Hasta hace poco se recolectaba información cuantitativa sobre indicadores tradicionales referidos a la eficiencia educativa entendida como la optimización que hace el sistema de los recursos económicos disponibles. Para saber que estaba funcionando bien bastaba observar los índices de matrícula, repetición, deserción, promoción, relación alumnos/profesor, costo alumno y otros que respondían a valorar el logro de coberturas y universalización de la educación. Actualmente los sistemas, más allá de metas expansivas, se plantean metas de calidad; se considera que dichos indicadores son insuficientes para valorar su eficacia, entendiéndose por ello los grados de cumplimiento de sus metas y objetivos educacionales propuestos y logros de competencias alcanzados tanto por el Sistema en su globalidad como por los estudiantes en determinados grados, ciclos o niveles, y la satisfacción que la sociedad en su conjunto tiene del servicio que brinda.

En este contexto, las universidades no están exentas de estas y otras preocupaciones. En la actualidad estas instituciones están implicadas en procesos de evaluación, en los que se suele proceder a un autoestudio seguido de una contrastación externa, cuyo primer objetivo explícito es la mejora de la calidad de las instituciones. Los procesos de mejora también se llevan a cabo a través de la implementación de modelos de dirección estratégica, de nuevas formas organizativas, de fórmulas de financiamiento ligadas a la calidad o mediante la adopción del enfoque de la calidad total 
(TEETER Y LOZIER, 1993; PEÑA, 1999; WINTER, 1999).

En Chile una de las principales transformaciones está relacionada con los cambios demográficos y de apertura de la universidad a la sociedad ya enfrenta un proceso de masificación y de generalización de la educación superior, se esta en presencia de un proceso de ampliación de la entrada de alumnos y a una cierta competencia por los estudiantes que van a entrar en la universidad, así como a la modificación de los perfiles de los nuevos alumnos.

Por otra parte, se detecta un cierto cuestionamiento de la funcionalidad y el rendimiento de las instituciones universitarias, junto con un incremento de las expectativas de la sociedad respecto a la actuación y los servicios de las universidades y una mayor exigencia de los diferentes usuarios de dichos servicios. Se observa asimismo una internacionalización gradual del sistema universitario y un aumento de la relación entre las universidades y muchas organizaciones públicas y privadas (VILALTA, 1999).

La universidad se encuentra con la necesidad de hacer frente a las nuevas demandas y necesidades sociales relacionadas con la calidad del sistema universitario, después de una etapa que se caracterizó por los objetivos de cantidad y acceso, así como la necesidad de potenciar sistemas transparentes de evaluación y garantía de la calidad. Las universidades deben hacer compatible su función tradicional de productoras y difusoras de conocimiento científico con las presiones por asumir nuevas tareas, atender nuevos usuarios y utilizar nuevos instrumentos.

Por todo ello, se observa actualmente en la universidad una preocupación por mantener y aumentar la calidad de la docencia, de la investigación y en general la de todos los servicios que presta. Las universidades han paulatinamente adoptado medidas de cambio y adaptación al nuevo entorno. Son de destacar, principalmente, los procesos de evaluación institucional introducidos por el Consejo de Educación Superior, La Comisión Nacional de Acreditación de Pregrado, la gestión de la calidad mediante la creación de vicerrectorados de desarrollo y otros soportes al proceso de mejora, y la planificación estratégica de las instituciones universitarias.

Esta preocupación por los aspectos relacionados con la calidad en las universidades ha abierto una línea de estudio en la que se encuadra esta investigación. Aunque hay varios enfoques sobre el concepto de la calidad universitaria, que no son excluyentes sino que hasta cierto punto vienen a 


\section{P. E. Rosales Villarroel}

ser complementarios, creemos que existe un enfoque, el de la calidad percibida, directamente vinculado a la calidad entendida como aptitud para satisfacer las necesidades de los usuarios.

Por otra parte, consideramos que faltan investigaciones rigurosas de cómo funcionan las iniciativas de calidad en la universidad y de cuáles son sus efectos, con el fin de obtener pruebas más concluyentes de la efectividad real de las acciones que se llevan a cabo para su mejora. En este sentido, esta investigación aborda diversos aspectos relacionados con los procesos de evaluación y mejora de la calidad universitaria, centrándose especialmente en el ámbito de la enseñanza y en un contexto específico, el de la Escuela de Pedagogía en Historia y Geografía de la Universidad Autónoma del Sur.

La investigación se realiza con el propósito de describir y reconocer desde la perspectiva de los actores el carácter particular de la gestión de la enseñanza en la Escuela de Pedagogía en Historia y Geografía de la Universidad Autónoma del Sur - Talca, y en sus relaciones con las demandas surgidas del contexto situacional y de las definiciones internas articuladas en el Proyecto Educativo Institucional.

En síntesis, se busca resolver la siguiente pregunta de investigación:

¿Qué características presenta la gestión de la calidad de la enseñanza en la Escuela de Pedagogía en Historia y Geografía de la Universidad Autónoma del Sur, desde la perspectiva de sus profesores y alumnos?

\section{Metodo / Modo de Indagación}

La orientación básica de este estudio se enmarca dentro del campo temático de la Teoría de la Organización Educativa, el cual se caracteriza por su gran amplitud y naturaleza multidisciplinar. Más concretamente, esta investigación se basa en las aportaciones que provienen especialmente del enfoque actual de la calidad. Aunque el estudio de la calidad está vinculado en sus inicios al área de producción, ha ido ampliando su incidencia hacia otros ámbitos de la actividad, dada su consideración de variable estratégica relevante susceptible de ser gestionada. En las últimas décadas los aspectos estratégicos de la calidad han sido reconocidos y puestos de manifiesto, con lo que se inicia la etapa actual de la Dirección de la Calidad, un aspecto dentro del contexto global de las Teorías Organizativas.

En otro orden, este trabajo se nutre de los fundamentos teóricos que 
provienen del campo temático de la Teoría de la Organización, el cual se basa en varias disciplinas científicas, entre las que destacamos la Economía, la Sociología, la Psicología y la Ciencia Política. En términos generales, la Teoría de la Organización abarca un gran número de perspectivas que tratan de comprender, explicar y predecir el comportamiento de las organizaciones. También se preocupa por el funcionamiento de las personas, los grupos y la estructura, investigando las repercusiones que todos ellos producen en el comportamiento de las organizaciones, con el propósito de aplicar estos conocimientos para mejorar la eficacia de una organización.

Los diferentes niveles básicos de análisis que pueden adoptarse en las organizaciones son los siguientes: individuo, grupo, organización y grupos de organizaciones. Para poder conocer, interpretar y mejorar con acierto el funcionamiento de las organizaciones es preciso poner de manifiesto la estrecha relación y la necesaria complementariedad de las perspectivas microorganizativas, que se ocupan de forma preferente del individuo y el grupo, y las macroorganizativas, que abordan con carácter prioritario la organización y los grupos de organizaciones.

En este sentido los objetivos de la investigación, radicada en el contexto antes descrito, se definen como sigue:

* Analizar los principales factores determinantes de la calidad de la enseñanza universitaria en la Escuela de Pedagogía en Historia y Geografía de la Universidad Autónoma del Sur, desde la perspectiva de sus actores, profesores y alumnos.

* Diferenciar enfoques sobre calidad de la enseñanza en relación al contexto de los sujetos que son parte de la investigación.

* Analizar los principales factores determinantes de la calidad de la enseñanza universitaria en la Escuela de Pedagogía en Historia y Geografía de la Universidad Autónoma del Sur, desde la perspectiva de sus actores, profesores y alumnos.

* Diferenciar enfoques sobre calidad de la enseñanza en relación al contexto de los sujetos que son parte de la investigación.

Los niveles o ámbitos de que consta la investigación son los siguientes:

A. Estudio de la calidad de servicio percibida por los estudiantes en el ámbito de la enseñanza universitaria, de las dimensiones que la conforman y de la influencia que tienen las características de los alumnos. 


\section{P. E. Rosales Villarroel}

B. Estudio de las actitudes del profesorado universitario (satisfacción laboral, autoeficacia y compromiso organizativo), de su relación con la calidad de la enseñanza percibida y de la influencia que tienen las características de los profesores.

En lo que respecta al primer nivel de investigación la evaluación de la calidad de la enseñanza se realiza únicamente a través de las percepciones de los estudiantes y por la vía de un cuestionario. Cabe tener presente que en el momento de realización del trabajo de campo parte de los encuestados había permanecido en la universidad por un tiempo mínimo de seis meses, lo que dificultaba la comparación entre expectativas y percepciones. Además, la elección de un enfoque basado sólo en las percepciones también presenta la ventaja que reduce la amplitud del cuestionario.

El proceso de investigación se desarrolló en dos grandes etapas. La primera fase tuvo como objetivo la identificación de-los atributos clave de la calidad de servicio en la enseñanza universitaria. En este sentido, la generación de los ítems que componen el cuestionario se basó en dos fuentes distintas de información:

A. La revisión de la literatura sobre calidad de servicio y calidad de la enseñanza, $y$

B. La realización de un estudio exploratorio previo sobre las formas de mejorar la calidad de la enseñanza desde el punto de vista del estudiante.

Respecto del segundo nivel de investigación, éste se desarrolló también en dos fases. La primera fase consistió en la elaboración del cuestionario a partir de la revisión de la literatura, y de las conclusiones de un estudio exploratorio previo (VECIANA Y CAPELLERAS, 2000). Se realizó un pretest de forma que el cuestionario elaborado se entregó a una serie de profesores para comprobar que la redacción y el significado de las preguntas eran adecuadas. Esta prueba se realizó con personal académico de la escuela en los que se llevó a cabo el trabajo de campo.

Como se observa la investigación se define metodológicamente como un estudio de caso. El estudio de casos es definido por Denny (1978, p. 370) como "un examen completo o intenso de una faceta, una cuestión o quizás los acontecimientos que tienen lugar en un marco geográfico a lo largo del tiempo". Otros autores como MacDonald y Walker (1977) hablan del estudio de casos como un examen de un caso en acción. Patton (1990) lo considera 
como una forma particular de recoger, organizar y analizar datos. Todas las definiciones vienen a coincidir en que el estudio de casos implica un proceso de indagación que se caracteriza por el examen detallado, comprehensivo, sistemático y en profundidad del caso objeto de interés (García Jiménez, 1991, p. 67).

La investigación se inscribe dentro de un modelo que integra elementos de orden cuantitativo, especialmente en la recogida de datos y elementos de orden cualitativo en la interpretación de los mismos. Lo anterior en la necesidad de configurar una realidad objetiva amplia en torno al diseño de un modelo que permita caracterizar y evaluar la gestión académica a partir de la descripción, interpretación y comprensión de la realidad de dicho componente de gestión y de los actores involucrados en ese mismo proceso.

\section{Conclusiones}

En términos de resultados se tiene que las valoraciones por parte de estudiantes y profesores de la calidad de la enseñanza a nivel de titulación, así como de los conocimientos, las actitudes y las capacidades del profesorado, del contenido de los estudios, de las instalaciones y equipamientos y de los sistemas de evaluación, no difieren excesivamente, aunque los estudiantes tienden en general a valoraciones inferiores de la mayor parte de estos aspectos.

Los resultados obtenidos dan cuenta de una alta valoración del nivel de conocimientos del profesorado, aspecto que resulta mejor valorado por ambos colectivos, y por otra los estudiantes puntúan peor los sistemas de evaluación del aprendizaje, mientras que los profesores valoran más negativamente las instalaciones y equipamientos.

Finalmente, para desarrollar un proceso de evaluación y acreditación de la calidad universitaria, es claro que ya no basta con una definición filosófica o académica de dicho concepto, es necesario definir un concepto de calidad con determinadas características que le permitan:

* Ser operacional, es decir poder traducirse en elementos fácilmente manejables dentro de una guía, modelo o procedimiento de evaluación;

* Que abarque de una u otra forma las funciones sustantivas de la universidad; 
* Que lleve implícito el concepto de apreciación o evaluación; y

* Que esté ligado a la pertinencia social.

\section{Referências}

ÁLVAREZ, M. y S. RODRÍGUEZ. "La calidad total en la Universidad: ¿Podemos hablar de clientes? Boletín de Estudios Económicos, vol. LII, 1997.

BUSCH, T., FALLAN, L. y A. PETTERSEN. Disciplinary differences in job satisfaction, self efficacy, goal commitment and organisational commitment in Norwegian Colleges: an empirical assessment of indicators of performance. Quality in Higher Education, 4, 1998.

CASANUEVA, C., PERIÁÑEZ, R. y J.E. RUFINO. Calidad percibida por el alumno en el servicio docente universitario: Desarrollo de una escala de medida. In: M. Ruiz (ed.), XI, Lleida: Congreso Nacional AEDEM, 1997.

DE MIGUEL, M. La evaluación de la enseñanza. Propuesta de indica dores para las titulaciones". In: VIDAL, J. (Coord.). Indicadores en la universidad: información y decisiones. Madrid: Consejo de Universidades, 1999.

DE MIGUEL, M. Utilización de indicadores en la evaluación de la docencia universitaria. In: DE MIGUEL, M., MORA, J.G. y S. RODRÍGUEZ. La evaluación de las instituciones universitarias. Madrid: Consejo de Universidades, 1991.

DENNY, T. Storytelling and educational understanding (Occasional Paper $\mathrm{N}^{\circ}$. 12). Kalamazoo: Western Michigan University, College of Education, 1978.

FERNÁNDEZ, Z. La estructura organizativa: Un análisis contingente. Investigaciones Económicas, vol. X, 1996.

GARCÍA JIMÉNEZ, E. Una teoría práctica sobre la evaluación. Estudio etnográfico. Sevilla. MIDO, 1991.

GONZÁLEZ ASTORGA, F.; GONZÁLEZ HERNANDO, S. y LI. ÁLVAREZ . Evaluación de los planes de estudio: Evidencias empíricas. In: M. Ruiz (Coord.), Lleida, XI Congreso Nacional AEDEM, vol. 2, 1997. 
MACDONALD, B.; WALKER R. Case-study and the social philosophy of educational research. In: Hamilton, D. (Eds.). Beyond the Numbers Game. London: Macmillan, 1977.

MINTZBERG, H. La estructuración de las organizaciones. Barcelona: Ariel, 1984.

MORA, J.G. Calidad y rendimiento en las instituciones universitarias. Madrid: Consejo de Universidades, 1991.

PATTON, M. Q. Qualitative evaluation and research methods (2nd ed.). Newbury Park, CA: Sage, 1990.

PEÑA, D. Calidad Total de las universidades. In: Comissionat per a Universitats i Recerca, Universitat: Estratégies per avançar. Barcelona: Dirección Estratégica y Calidad en las Universidades, UPC, 1999.

PÉREZ, C. y J. SALINAS. El uso de indicadores de gestión en la evaluación de la calidad universitaria”, Hacienda Pública Española, núm. especial sobre Economía de la Educación, 1998.

RODRÍGUEZ ESPINAR, S. La evaluación institucional universitaria. Revista de Investigación Educativa, 1997.

RODRÍGUEZ ESPINAR, S. La investigación en evaluación institucional. Cuadernos IRC, 1999.

TAN, D.L. Evaluación de la calidad de la enseñanza superior: una revisión de la bibliografía y la investigación, In: J.G. MORA, M. DE MIGUEL y S. RODRÍGUEZ (eds.). La evaluación de las instituciones universitarias. Madrid: Consejo de Universidades, p. 165-21, 1991.

VECIANA, J.M. La nueva estructura matricial de la Universidad. La Vanguardia, 26/04, 1995.

VECIANA, J.M. y CAPELLERAS, J.L. La mejora de la docencia y del rendimiento académico", ponencia presentada en el 1er. Congreso Internacional Docencia Universitaria e Innovación, 26-28 junio, Barcelona, 2000 .

WINTER, R.S. El viaje de la calidad: Lo que he visto, oído y aprendido. In: Comissionat per a Universitats i Recerca, Universitat: Estratégies per avançar. Dirección Estratégica y Calidad en las Universidades, UPC, 
Pedro Enrique Rosales Villarroel

Doutor em Ciências da Educação, Diretor da Escola de Educação da Universidade Autônoma do Chile em Talca, Região do Maule, Chile.

E-mail para correspondência:

prosalesv@uautonoma.cl 